\title{
Novel bacterial topoisomerase inhibitors: challenges and perspectives in reducing hERG toxicity
}

\author{
Anja Kolarič ${ }^{1,2} \&$ Nikola Minovski*,1 \\ ${ }^{1}$ Theory Department, Laboratory for Cheminformatics, National Institute of Chemistry, Hajdrihova 19, 1000 Ljubljana, Slovenia \\ ${ }^{2}$ Department of Pharmaceutical Chemistry, Faculty of Pharmacy, University of Ljubljana, Aškerčeva 7, 1000 Ljubljana, Slovenia \\ *Author for correspondence: Tel.: +386 14760 383; Fax.: +386 14760 300; nikola.minovski@ki.si;
}

First draft submitted: 26 June 2018; Accepted for publication: 7 August 2018; Published online: 14 September 2018

Keywords: bacterial DNA gyrase • intercalating antibacterial agents $\bullet$ NBTIs optimization • novel bacterial topoisomerase inhibitors $\bullet$ reducing hERG toxicity $\bullet$ structure-activity relationship

Regardless of the long term therapeutic success of quinolones in antibacterial chemotherapy, new forms of quinolones-induced resistance in bacterial pathogens are constantly arising indicating a decreased or even complete ineffectiveness of these drugs in antibacterial treatments [1]. In the quest for innovative antibacterial alternatives targeting bacterial topoisomerases II replication/transcription machineries, novel bacterial topoisomerase inhibitors (NBTIs) representing an utterly new class of antibacterials have recently been discovered $[2,3]$. A remarkable breakthrough in the NBTIs development was accomplished when GlaxoSmithKline (GSK) revealed the very first $14 \mathrm{nM}$ NBTI (GSK299423) in complex with DNA gyrase originating from Staphylococcus aureus solved by $\mathrm{x}$-ray crystallography [4], which elevated NBTIs-related R\&D to a significantly higher, structure-based level [5]. In the last decade, the efforts of numerous medicinal chemists yielded various highly optimized NBTIs demonstrating remarkable broad-spectrum antibacterial potency against the most problematic Gram-positive and Gram-negative pathogens. Notwithstanding their notable nanomolar antibacterial activity and potency firmly confirmed in in vitro/in vivo assays, a key issue that unfortunately is still accompanying these antibacterials is their relatively high cardiotoxic potential denoted as hERG toxicity [6]. Consequently, a number of progressive NBTIs were discontinued from clinical trials. In the following text we give a brief overview of the current state-of-the-art NBTIs optimization protocols aiming to reduce their cardiotoxic potential, while retaining and/or improving their antibacterial properties.

\section{SAR-hERG profiling of NBTIs}

Disregarding the well-established cornerstones in the rational development of NBTIs as demonstrated by the remarkably active GSK299423 lead [4], its hERG liability might be the major obstacle for its progression as demonstrated by practically no published further data on its development. Instead, GSK thoroughly explored any bioisosteric replacements of the protonated amine (a part of the piperidine linker moiety) and their potential impact on reducing GSK299423-induced cardiotoxicity; hence, the aminopiperidine linker moiety was found as the most optimal one [5] and all cardinal modifications toward reducing hERG toxicity were performed on left-hand side (LHS) and right-hand side (RHS) fragments.

In 2013, GSK revealed a novel series of (S)-hydroxyl tricyclic NBTIs as exemplified by the GSK966587 lead (an naphthyridine-assembled tricyclic LHS-based NBTI) with high potency against Gram-positive and Gram-negative pathogens and acceptable hERG profile $\left(\mathrm{IC}_{50}=310 \mu \mathrm{M}\right)$ [7]. With the intention to further improve hERG, various LHS alterations were performed including introduction of quinoline, quinoxaline, double-fluorinated naphthyridine LHS, and even removal or replacement of the bridgehead tricyclic hydroxyl with fluorine. However, despite the good antibacterial potency of the resulting NBTI variants, all these LHS alterations have negative impact on hERG $\left(\mathrm{IC}_{50}=122-206 \mu \mathrm{M}\right)$.

Although the quinoline variant of GSK966587 did not perform well, the lead optimization efforts invested by the same GSK group yielded a novel series of non-hydroxyl quinoline-based tricyclic NBTIs governed by GSK945237 lead [8]. While this NBTI demonstrates excellent antibacterial properties (e.g., DNA gyrase/topo IV dual targeting) 
and relatively good hERG $\left(\mathrm{IC}_{50}=238 \mu \mathrm{M}\right)$, its quinoxaline LHS variant dramatically increases hERG toxicity $\left(\mathrm{IC}_{50}=48 \mu \mathrm{M}\right)$.

It seems that the utilization of tricyclic LHS moieties could be a good starting point for development of other broad-spectrum NBTI variants. In 2017, RedX Pharma launched their own NBTI series based on tricyclic LHSs comprised of fluorooxazoloquinolinone, fluoroisooxazoloquinolinone, methoxyisoxazoloquinolinone, and isoxazolonaphthyridinone building-blocks, with good antibacterial potency against Gram-negative Escherichia coli and good (e.g., $\mathrm{IC}_{50}>100 \mu \mathrm{M}$; isoxazolonaphthyridinones) to moderate (e.g., $\mathrm{IC}_{50}>33 \mu \mathrm{M}$; oxazoloquinolonones/isoxazoloquinolinones) hERG inhibitory profile [9]. With the aim to improve their hERG liability, the pyridoxazinecarbaldehyde RHS fragment was modified by fluorination, however, this strongly increases hERG toxicity $\left(\mathrm{IC}_{50} \sim 8-9 \mu \mathrm{M}\right)$.

Supposedly, the inclusions of substituted-LHS naphthyridine moieties have some potential in tuning NBTIs hERG profile. Apparently, this was also recognized by Actelion Pharmaceuticals Ltd., reporting an attractive series of dual targeting NBTI constructs substantiated on tetrahydropyran central unit linked to naphthyridine/quinoxaline LHS through syn-diol chain [10]. Interestingly, it was found that retaining/removing the 8-N-atom in both LHSs improves hERG (e.g., $\sim 50 \%$ ) and decreases the antibacterial potency, while fluorination at position 2 is not impacting hERG. More importantly, the introduction of oxathiinopyridine RHS gives not only high in vitro antibacterial potency against $S$. aureus $\left(\mathrm{IC}_{50}=30 \mathrm{nM}\right.$ ) comparable to GSK299423 [4], but also a satisfactory hERG toxicity profile (e.g., 19\% hERG blockage at $10 \mu \mathrm{M}$ ). Although improved activity on Gram-negative bacteria was achieved by replacing the hydroxyl group of the linker chain with amine, which yielded more polar compounds with additional positive charge, the magnitude of hERG blocking negatively contributes towards further development of this NBTI series [11].

In 2013, Pfizer introduced a novel series of dual targeting DNA gyrase/topo IV quinoline-LHS, butylphenyl RHS-based NBTIs as typified by the representative 3,6-difluorophenyl variant [12]. This lead showed excellent in vitro antibacterial potency against $S$. aureus, however, it was found to be strongly cardiotoxic as exemplified by its hERG blocking $\left(\mathrm{IC}_{50}=9.97 \mu \mathrm{M}\right)$. A similar hERG outcome was also perceived in case of 2-fluorophenyl, 3-fluorophenyl and 2,5-difluorophenyl RHS variants, while slight hERG improvements were noticed for 2,6-difluorophenyl and 2,3,5-trifluorophenyl RHS analogs $\left(\mathrm{IC}_{50}=47.6\right.$ and $30.9 \mu \mathrm{M}$, respectively). The utilization of 3-fluoroquinoline or 3-fluoro-1,5-naphthyridine LHS in combination with 2,5-difluorophenyl RHS gives equipotent NBTI analogs with moderate hERG-blocking affinities ( $\mathrm{IC}_{50}=85.9$ and $45 \mu \mathrm{M}$, respectively). An incomparably better hERG profile was achieved for 3-fluoroquinoline LHS-based NBTIs by using a more polar isoxazole RHS $\left(\mathrm{IC}_{50}>\right.$ $300 \mu \mathrm{M}$ ), unfortunately for the price of loosing its antibacterial potency [13].

\section{Impact of structure-lipophilicity-basicity triade on hERG}

In the pursuit of developing potent NBTIs with an acceptable hERG toxicity profile, AstraZeneca implemented a rational optimization approach grounded on reduction and further fine tuning between $\mathrm{pKa}$ and $\log \mathrm{D}$ [14]. The isosteric replacement of the piperidine linker with trans-aminoethyl cyclohexyl connected to 7methoxyquinoxalinone/naphthyridinone LHS can significantly reduce $\mathrm{pKa}$, while the inclusion of a second basic group at 2-position of the ethyl bridge can lower $\log \mathrm{D}$, resulting in improved hERG $\left(\mathrm{IC}_{50}>333 \mu \mathrm{M}\right)$. Conversely, 7-fluoronaphthyridinone LHS gives a fairly good hERG profile $\left(\mathrm{IC}_{50}>100 \mu \mathrm{M}\right)$, however this is followed by a drop in the antibacterial potency.

The positive trend between detracting overall $\log \mathrm{D}$ of NBTIs and consequently their hERG blocking affinity was also ascertained by Merck Pharmaceuticals, which introduced a series of oxabicyclooctane-based NBTIs as typified by the exemplar lead AM-8085 [15]. Notwithstanding its broad antibacterial spectrum, AM-8085 was found to be a strong hERG blocker $\left(\mathrm{IC}_{50}=0.6 \mu \mathrm{M}\right)$ that opened the avenue towards the rational hERG optimization.

A slight, but unsatisfactory hERG improvement was accomplished by increasing the polarity through attaching a hydroxyl group at $\mathrm{C}-2$ of the linker chain $\left(\mathrm{IC}_{50}=18 \mu \mathrm{M}\right.$; AM-8191) [15], while the alteration of 1,5-naphthyridine LHS to $(S)$-hydroxyl tricyclic naphthyridinone significantly ameliorates hERG $\left(\mathrm{IC}_{50}=394 \mu \mathrm{M}\right)$ [16]. Moreover, pyridoxazinone and pyridodioxane RHS fragments seem suitable in all respects; the alteration of the ring oxygen in both RHSs with sulfur is well tolerated for activity, however this increases hERG toxicity $\left(\mathrm{IC}_{50}=25.7 \mu \mathrm{M}\right)$. In particular, pyridodioxane RHS highly improves hERG $\left(\mathrm{IC}_{50}=764 \mu \mathrm{M}\right)$, unfortunately this tremendously diminishes the antibacterial potency and spectrum [17].

Interestingly, the transformation of the protonated amine at the C-7 position of the linker chain (a key attachment point for NBTIs antibacterial activity) to amide does not affect antibacterial potency and spectrum, however strongly 
reduces functional hERG activity $\left(\mathrm{IC}_{50} \geq 30 \mu \mathrm{M}\right)$ [18]. Moreover, the isosteric replacement of currently known linkers with a trans-dioxane scaffold seems to additionally contribute to the overall improved NBTIs hERG safety profile by reducing basicity and increasing polarity [19].

\section{Conclusion}

In a decade of intensive research, NBTIs undoubtedly prove their extraordinary potential as future agents for antibacterial chemotherapy. Indeed, despite their excellent antibacterial properties, NBTIs-induced hERG toxicity still remains a pivotal encumbrance for their advancement into clinical trials. It seems that regardless of the different NBTIs rational optimizations invested for improving hERG, they negatively impact the overall biological activity and vice versa. The fine-tuning of NBTIs physicochemical properties (e.g., $\log \mathrm{D}, \mathrm{pKa}$ ) could offer a promising solution towards diminishing hERG-related issues, while retaining antibacterial potencies.

\section{Financial \& competing interests disclosure}

The authors thank the Agency of Research of R Slovenia (ARRS) for financial support through the Grant P1-0017. The authors have no other relevant affiliations or financial involvement with any organization or entity with a financial interest in or financial conflict with the subject matter or materials discussed in the manuscript apart from those disclosed.

No writing assistance was utilized in the production of this manuscript.

\section{References}

1. Jacoby GA. Mechanism of resistance to quinolones. Clin. Infect. Dis. 41(Suppl. 2), S120-S126 (2005).

2. Gomez L, Hack MD, Wu J et al. Novel pyrazole derivates as potent inhibitors of type II topoisomerases. Part 1: synthesis and preliminary SAR analysis. Bioorg. Med. Chem. Lett. 17(10), 2723-2727 (2007).

3. Wiener JJM, Gomez L, Venkatesan H et al. Tetrahydroindazole inhibitors of bacterial type II topoisomerases. Part 2: SAR development and potency against multidrugresistant strains. Bioorg. Med. Chem. Lett. 17(10), 2718-2722 (2007).

4. Bax BD, Chan PF, Eggleston DS et al. Type II topoisomerase inhibiton by a new class of antibacterial agents. Nature 466(19), 935-940 (2010).

5. Widdowson K, Hennessy A. Advances in structure-based drug design of novel bacterial topoisomerase inhibitors. Future Med. Chem. 2(11), 1619-1622 (2010).

6. Black MT, Stachyra T, Patel D et al. Mechanism of action of the antibiotic NXL101, a novel non-fluoroquinolone inhibitor of bacterial type II topoisomerases. Antimicrob. Agents Chemother. 52(9), 3339-3349 (2008).

7. Miles TJ, Hennessy AJ, Bax B et al. Novel hydroxyl tricyclics (e.g., GSK966587) as potent inhibitors of bacterial type IIA topoisomerases. Bioorg. Med. Chem. Lett. 23, 5437-5441 (2013).

8. Miles TJ, Hennessy AJ, Bax B et al. Novel tricyclics (e.g., GSK945237) as potent inhibitors of bacterial type IIA topoisomerases. Bioorg. Med. Chem. Lett. 26, 2464-2469 (2016).

9. Charrier C, Salisbury A-M, Savage VJ et al. Novel bacterial topoisomerase inhibitors with potent broad-spectrum activity against drug-ressistant bacteria. Antimicrob. Agents Chemother. 61(5), e02100-e02116 (2017).

10. Surivet J-P, Zumbrunn C, Rueedi G et al. Design, synthesis, and characterization of novel tetreahydropyran-based bacterial topoisomerase inhibitors with potent anti-Gram-positive activity. J. Med. Chem. 56, 7396-7415 (2013).

11. Surivet J-P, Zumbrunn C, Bruyère $\mathrm{T}$ et al. Synthesis and characterization of tetrahydropyran-based bacterial topoisomerase inhibitors with antibacterial activity against Gram-negative bacteria. J. Med. Chem. 60, 3776-3794 (2017).

12. Mitton-Fry MJ, Brickner SJ, Hamel JC et al. Novel quinoline derivatives as inhibitors of bacterial DNA gyrase and topoisomerase IV. Bioorg. Med. Chem. Lett. 23, 2955-2961 (2013).

13. Mitton-Fry MJ, Brickner SJ, Hamel JC et al. Novel 3-fluoro-6-methoxyquinoline derivatives as inhibitors of bacterial DNA gyrase and topoisomerase IV. Bioorg. Med. Chem. Lett. 27, 3353-3358 (2017).

14. Reck F, Ehmann DE, Dougherty TJ et al. Optimization of psysicochemical properties and safety profile of novel bacterial topoisomerase type II inhibitors (NBTIs) with activity against Pseudomonas aeruginosa. Bioorg. Med. Chem. 22, 5392-5409 (2014).

15. Singh SB, Kaelin DE, Wu J et al. Oxabicyclooctane-linked novel bacterial topoisomerase inhibitors as broad spectrum antibacterial agents. ACS Med. Chem. Lett. 5, 609-614 (2014).

16. Singh SB, Kaelin DE, Wu J et al. Tricyclic 1,5-naphthyridinone oxabicyclooctane-linked novel bacterial topoisomerase inhibitors as broad-spectrum antibacterial agents-SAR of left-hand-side moiety (Part-2). Bioorg. Med. Chem. Lett. 25, 1831-1835 (2015).

17. Singh SB, Kaelin DE, Wu J et al. Hydroxy tricyclic 1,5-naphthyridinone oxabicyclooctane-linked novel bacterial topoisomerase inhibitors as broad-spectrum antibacterial agents-SAR of RHS moiety (Part-3). Bioorg. Med. Chem. Lett. 25, 2473-2478 (2015). 
18. Singh SB, Kaelin DE, Wu J et al. Structure activity relationship of pyridoxazinone substituted RHS analogs of oxabicyclooctane-linked 1,5-naphthyridinyl novel bacterial topoisomerase inhibitors as broad-spectrum antibacterial agents (Part-6). Bioorg. Med. Chem. Lett. 25, 3636-3643 (2015).

19. Li L, Okumu A, Dellos-Nolan S et al. Synthesis and anti-staphylococcal activity of novel bacterial topoisomerase inhibitors with a 5-amino-1,3-dioxane linker moiety. Bioorg. Med. Chem. Lett. doi: 10.1016/j.bmcl.2018.06.003 (2018) (Epub ahead of print). 